Universidade Tecnológica Federal do Paraná - UTFPR

Campus Ponta Grossa - Paraná - Brasil

ISSN: 1981-3686 / v. 03, n. 01: p. 70-76, 2009
Revista Brasileira deTecnologia

Agroindustrial

\title{
EFEITO DA GOMA XANTANA NAS CARACTERÍSTICAS SENSORIAIS DE BOLOS SEM GLÚTEN
}

\section{EFFECT OF THE XANTHAN GUM IN THE SENSORY CHARACTERISTIC OF GLUTEN FREE CAKES}

\author{
Leidi D. Preichardt ${ }^{1}$; Claire T. Vendruscolo ${ }^{2}$; Márcia A. Gularte ${ }^{3}$; Angelita da S. Moreira ${ }^{4}$ \\ ${ }^{1}$ Departamento de Ciência e Tecnologia Agroindustrial - Universidade Federal de Pelotas - UFPel \\ - Pelotas - Brasil preichardt leidi@yahoo.com.br \\ ${ }^{2,3,4}$ Departamento de Ciência dos Alimentos - Universidade Federal de Pelotas - UFPel - Pelotas - \\ Brasil claire@ufpel.tche.br
}

\begin{abstract}
Resumo
O objetivo deste trabalho foi avaliar o efeito da goma xantana nas características sensoriais de bolos sem glúten feitos com farinhas de arroz e milho. Quatro formulações foram testadas: (F1) controle - sem xantana, (F2) com 0,2\% de xantana, (F3) com 0,3\% de xantana e (F4) com 0,4\% de xantana. As formulações foram elaboradas com farinha de arroz, de milho, açúcar, leite, ovos, óleo de soja, xantana e fermento químico. A massa foi preparada, colocada em formas e assada a $200^{\circ} \mathrm{C}$ por 35 minutos. A caracterização sensorial foi realizada com 13 julgadores treinados que avaliaram nove atributos do bolo: cor do miolo, formação de migalhas, elasticidade, firmeza, porosidade, sabor de arroz, sabor de milho, sensação de umidade na boca e envelhecimento; esta caracterização foi realizada com bolos frescos e armazenados durante 3, 6 e 9 dias. Para a realização desta etapa foi utilizada uma escala não estruturada de $9 \mathrm{~cm}$. A adição de xantana melhorou as características sensoriais dos bolos, marcadamente retardando o envelhecimento, reduzindo a formação de migalhas e aumentando a sensação de umidade na boca, sendo que as amostras com $0,3 \%$ e $0,4 \%$ de xantana tiveram as melhores características. Portanto, a goma xantana pode ser utilizada de maneira satisfatória na produção de bolos isentos de glúten, melhorando expressivamente as características e, assim, sua qualidade sensorial.
\end{abstract}

Palavras-chave: xantana, bolo sem glúten; farinha de arroz; farinha de milho; análise sensorial.

\section{Introdução}

Os produtos de panificação estão presentes no dia-a-dia dos brasileiros e representam um faturamento anual ao redor de R $\$ 25$ bilhões, além de gerar cerca de 550 mil empregos diretos. $\mathrm{O}$ setor é composto por 100 mil pequenos empresários em 52 mil empresas (SINDIPAN, 2008). 
Produtos de panificação têm como principal ingrediente a farinha de trigo devido as suas características reológicas. Estas características são atribuídas ao glúten, que é um conjunto de proteínas (glutenina e gliadina) presente no trigo, aveia, centeio, cevada e malte. Alguns indivíduos, no entanto, apresentam uma predisposição genética que faz com que o intestino delgado apresente uma intolerância permanente ao glúten, ficando assim impedidos de ingerir alimentos, bebidas e medicamentos que contenham esta substância. A doença atinge cerca de 1 adulto a cada 474 e cerca de 1 criança a cada 169 (Silva, 2007; Sivaramakrishnan, et al, 2004).

Desta forma, os cientistas de alimentos tentam buscar ingredientes que apresentem características funcionais semelhantes ao glúten, sem prejuízo à qualidade dos alimentos e à saúde dos consumidores. A substituição do glúten em produtos de panificação, no entanto, é um grande desafio, pois ele é o responsável pela extensão e elasticidade da massa, retendo o ar e dando volume aos produtos.

O arroz vem sendo uma ótima opção como substituto do trigo na produção de farinhas utilizadas em produtos de panificação. A farinha de arroz é nutritiva e, por não possuir o glúten, pode ser utilizada por pessoas portadoras da doença celíaca. O milho também é um cereal possível de ser utilizado para este fim. No entanto, ambos apresentam capacidade limitada para reter o ar (Galera, J., 2006).

A goma xantana é um hidrocolóide polissacarídico que possui excelentes propriedades reológicas de interesse para a utilização em alimentos. Auxilia na retenção de gás e no aumento do volume específico dos produtos de panificação e por apresentar estas características é objetivo de estudo de diversos pesquisadores (Collar, et. al 1999; Guarda, et. al. 2004; Lazaridou, A. et.al.2007; Tubari, et.al.,2008). Tubari et al. (2008) avaliaram as propriedades reológicas e a qualidade de bolos de arroz formulados com diferentes gomas (xantana, guar, locusta, $\kappa$-carragena, hidroxi-propilmetil-celulose, mistura de xantana-guar, mistura de xantana- $\kappa$-carragena) e uma mistura de emulsificantes. Nesse estudo as massas dos bolos feitos com xantana e a mistura de xantana-guar tiveram os maiores valores de viscosidade aparente. Das formulações sem emulsificantes o bolo produzido com a xantana obteve maior volume específico. Esses resultados demonstram uma estabilidade da massa e qualidade do bolo produzido com xantana.

A farinha de arroz tem um sabor pouco pronunciado, embora confira uma textura arenosa e sensação de secura, e sua mistura com a farinha de milho é uma forma eficaz de incrementar o sabor da mistura de forma natural e sem necessidade do uso de aditivos. As farinhas de arroz e milho juntamente com a xantana podem ser utilizadas para a obtenção de bolos sem glúten com características aceitáveis. 
Este trabalho teve por objetivo estudar o efeito da aplicação da goma xantana nas características sensoriais de bolos sem glúten formulados com farinha de arroz e farinha de milho.

\section{Material e Métodos}

Para o preparo das formulações foram utilizadas farinha de milho, farinha de arroz, goma xantana, açúcar refinado, ovos, óleo de soja, fermento químico e leite bovino pasteurizado.

Quatro formulações foram testadas: (C1) controle, sem a goma xantana; (F1) com 0,2\% de goma xantana, (F2) com $0,3 \%$ de goma xantana e (F3) com $0,4 \%$ de goma xantana. As concentrações de goma xantana foram calculadas com base no peso final da massa após a adição de todos os ingredientes. As massas foram preparadas e acondicionadas em formas untadas e padronizadas para as quatro formulações e imediatamente colocadas em forno pré-aquecido a $200^{\circ} \mathrm{C}$ por 35 minutos. Após esfriar, os bolos foram desenformados, acondicionados em sacos de polietileno tratados com solução de metilparabeno $0,2 \%$ e armazenados na temperatura ambiente.

Para a caracterização dos bolos foi utilizado um painel com 13 julgadores treinados, homens e mulheres, que avaliaram nove atributos: cor do miolo, migalhas formação, elasticidade, firmeza, porosidade, sabor de arroz, sabor de milho, sensação de umidade na boca e envelhecimento, mostrados na Tabela 1. As avaliações foram realizadas no mesmo dia da preparação (T0) e após 3 (T3), 6 (T6) e 9 (T9) dias de armazenamento. A escala não estruturada de 9 centímetros foi utilizada para a avaliação. O lado esquerdo da escala correspondia à menor intensidade (valor 0,0 ) e o lado direito à maior intensidade (valor 9,0). As avaliações foram realizadas no laboratório de análise sensorial da Universidade Federal de Pelotas (UFPel, Pelotas, Brasil). As quatro amostras foram apresentadas sob a forma monádica seqüencial em temperatura ambiente, com porções de $20 \mathrm{~g}$ por amostra e servidas em recipientes codificados com três dígitos aleatórios. Foi solicitado que entre uma e outra amostra o palato fosse lavado com água.

\section{Resultados e Discussão}

Os gráficos aranha, ilustrados na Figura 1 sugerem que todas as amostras, independentemente da formulação e do tempo de armazenamento, foram semelhantes quanto aos atributos sabor de arroz, sabor de milho, porosidade e cor do miolo. A formulação base, combinando farinha de arroz à de milho, mostrou-se eficiente em mascarar o gosto e textura, principalmente, característico de arroz. Os bolos frescos adicionados de xantana promoveram uma sensação de umidade na boca maior quando comparado com o bolo controle (C1) sem xantana. A 
formulação (C1), mostrou menor elasticidade, maior formação de migalhas e firmeza, em todos os tempos, além de envelhecimento já a partir do terceiro dia de armazenamento. Esta formulação, portanto, caracterizou-se por apresentar um envelhecimento muito superior às demais formulações, principalmente no nono dia de armazenamento. A adição de xantana promoveu maior estabilidade da massa em relação ao tempo de armazenamento e melhorou sensorialmente o bolo formulado com farinha de arroz e milho. Sensorialmente, as formulações que tiveram as melhores características foram (3) e (4), ou seja, as formulações com as maiores concentrações xantana, 0,3\% e 0,4\% respectivamente.

Tabela 1- Definição dos atributos sensoriais

\begin{tabular}{|c|c|}
\hline Atributos Sensoriais & Definição \\
\hline \multirow[t]{3}{*}{ Cor do miolo } & Cor amarela \\
\hline & Sem intensidade $=$ amarela clara \\
\hline & Alta intensidade $=$ amarela escura \\
\hline \multirow[t]{3}{*}{ Formação de migalhas } & Formação de migalhas quando cortado ou apertado. \\
\hline & Sem intensidade $=$ pouca formação de migalha quando corta ou aperta \\
\hline & Alta intensidade $=$ alta formação de migalhas quando corta ou aperta \\
\hline \multirow[t]{3}{*}{ Elasticidade } & Os pedaços são capazes retornar a forma original depois de apertar \\
\hline & $\begin{array}{l}\text { Sem intensidade = não é elástico, pedaços não voltam a forma original } \\
\text { depois de apertar }\end{array}$ \\
\hline & $\begin{array}{l}\text { Alta intensidade }=\text { muito elástico, os pedaços retornam a forma original } \\
\text { depois de apertar }\end{array}$ \\
\hline \multirow[t]{3}{*}{ Firmeza } & Força necessária para morder \\
\hline & Sem intensidade $=$ pouca força necessária para morder \\
\hline & Alta intensidade $=$ muita força necessária para morder \\
\hline \multirow[t]{3}{*}{ Porosidade } & Estrutura dos poros no miolo \\
\hline & Desuniforme $=$ poros aleatórios de diversos tamanhos \\
\hline & Uniforme $=$ densa estrutura de poros \\
\hline \multirow[t]{3}{*}{ Sabor de arroz } & Sabor percebido de arroz \\
\hline & Sem intensidade $=$ sabor de arroz pouco percebido \\
\hline & Alta intensidade $=$ sabor de arroz muito percebido \\
\hline \multirow[t]{3}{*}{ Sabor de milho } & Sabor de milho percebido \\
\hline & Sem intensidade $=$ pouco sabor de milho percebido \\
\hline & Alta intensidade $=$ muito sabor de milho percebido \\
\hline \multirow{3}{*}{$\begin{array}{l}\text { Sensação de umidade na } \\
\text { boca }\end{array}$} & Sensação de umidade na boca \\
\hline & Sem intensidade $=$ seco, sem fluidez \\
\hline & Alta intensidade $=$ úmido, muita fluidez \\
\hline \multirow[t]{3}{*}{ Envelhecimento } & Envelhecimento \\
\hline & Sem intensidade $=$ nada de envelhecimento \\
\hline & Alta intensidade $=$ muito velho \\
\hline
\end{tabular}


Figura 1- Gráficos dos atributos avaliados durante 0,3,6 e 9 dias de armazenamentos

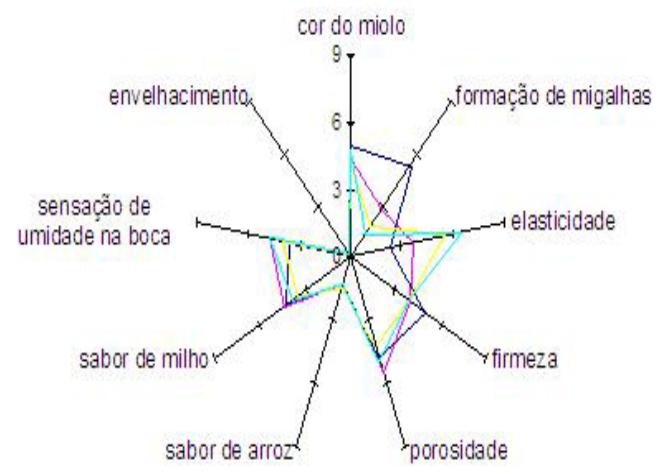

Tempo 0

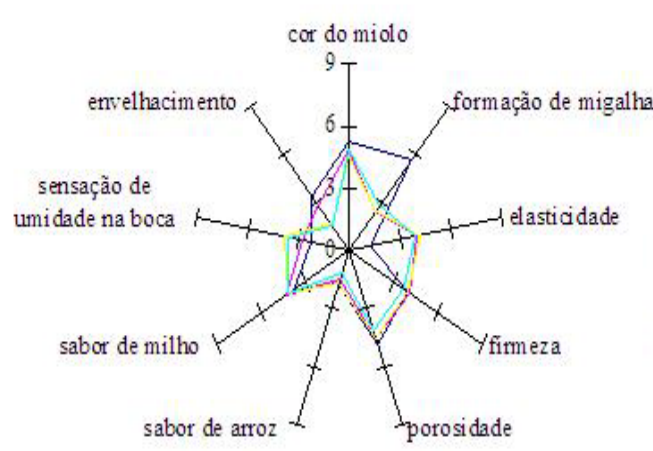

Tempo 6

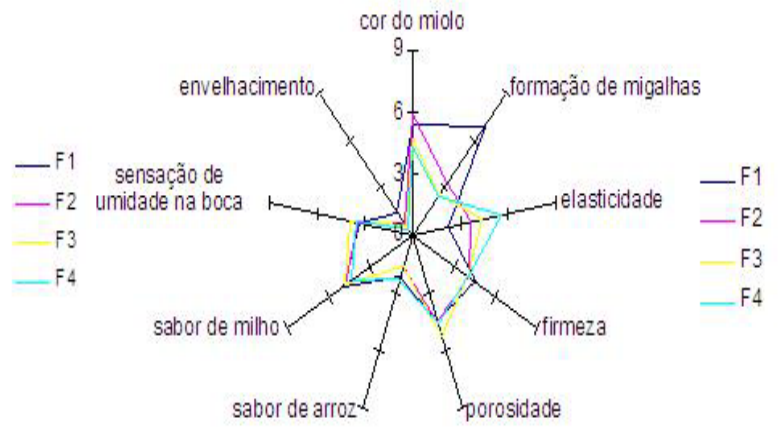

Tempo 3

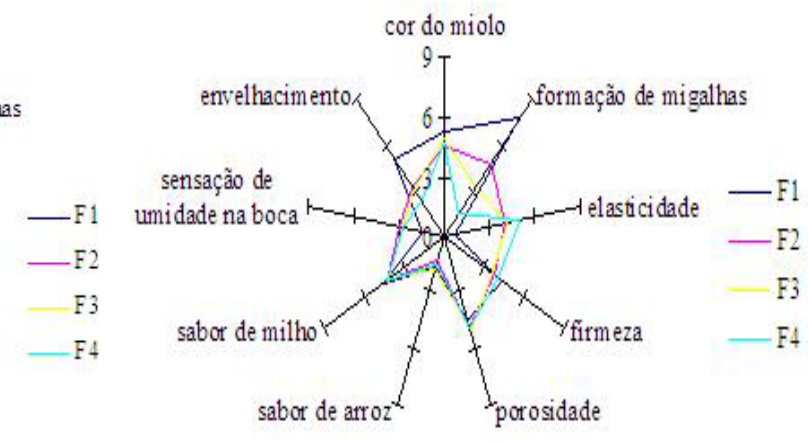

Tempo 9

\section{Conclusão}

A adição de xantana melhorou as características sensoriais dos bolos sem glúten formulados com farinha de arroz e milho. O retardo do envelhecimento, a menor formação de migalhas e a maior sensação de umidade na boca se destacaram nos bolos adicionados desta goma. Portanto, a goma xantana teve efeito benéfico sobre as características sensoriais dos bolos sem glúten podendo ser utilizada de maneira satisfatória na elaboração desses produtos, melhorando expressivamente sua qualidade sensorial.

\section{Abstract}

The aimed of this work was to evaluate the effect of the xanthan gum in the sensory characteristics of cakes without gluten done with rice and corn flours. Four formulations were tried: (F1) control without xanthan, (F2) with $0,2 \%$ of xanthan, (F3) with $0,3 \%$ of xanthan and (F4) with $0,4 \%$ of xanthan. The formulations were elaborated with rice and corn flour, sugar, milk, eggs, soya oil, 
xanthan and baking powder. The mass was ready, placed in forms and baked to $200^{\circ} \mathrm{C}$ for 35 minutes. The cakes sensory characterization was accomplished with 13 trained panelist that evaluated nine attributes: color of the crumb, crumbs formation, elasticity, firmness, porosity, rice flavor, corn flavor, sense of moisture in the mouth and staling. This characterization was accomplished in the fresh cakes and with 3,6 and 9 days of storage. For the evaluation of this stage we used a not-structured scale of $9 \mathrm{~cm}$. The xanthan addition improved the sensory characteristics of the cakes, remarkably retarding the aging, reducing the crumbs formation and increasing the moisture sensation in the mouth. The sample with $0,3 \%$ and $0,4 \%$ of xanthan introduced the best sensory characteristics. Thence xanthan gum can be used of satisfactory way in the gluten exempt cakes production, improving expressively the characteristics and, this way, its sensory quality.

Key-words: xanthan gum in cake gluten-free; rice flour; corn flour; sensory.

\section{Referências}

COLLAR, C.; ANDREU, P.; MARTINEZ, J. C.; ARMERO, E. Optimization of hydrocolloid addition to improve wheat bread dough functionality: a response surface methodology study. Food Hydriocolloids. v. 13, p. 467-475, 1999.

GALERA, J. Substituição parcial da farinha de trigo por farinha de arroz (Oryza sativa L.) na produção de “sonho" - estudo modelo. Dissertação - Mestrado em Ciência dos Alimentos. USP. São Paulo. 2006. 99p.

GUARDA, A.; ROSELL, C. M.; BENEDITO, C.; GALOTTO, M. J. Different hydrocolloids as bread improvers and antistaling agents. Food Hydrocolloids . v. 18, p. 241-247, 2004.

LARAZIDOU, A.; DUTA, D.; PAPAGEORGIOU, M.; BELE, N.; BILIADERIS, G. G. Effects of hydrocolloids on dough rheology and bread quality parameters in gluten-free formulations. Journal of Food Engineering, v. 79, p. 1033-1047, 2007.

SILVA, P. R. Restrições alimentares na alimentação escolar - doença celíaca. In: Encontro regional de nutricionistas da alimentação escolar da região centro-oeste, 2007.

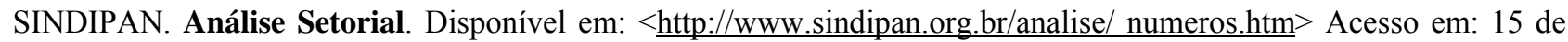
abril de 2008.

SIVARAMAKRISHNAN, H. P.; SENGE, B.; CHATTOPADHYAY, P. K. Rheological properties of rice dough for making rice bread. Journal of Food Engineering. v. 62, p. 37-45, 2004.

TUBARI, E.; SUMNU, G.; SAHIN, S. Rheological properties and quality of rice cakes formulated with different gums and an emulsifier blend. Food Hidrocolloids. V. 22, p 305-312, 2008.

\section{Inserir aqui dados completos de todos os autores:}

Nome completo: Leidi D. Preichardt

Filiação institucional: Universidade Federal de Pelotas

Função ou cargo ocupado: Mestranda em Ciência e Tecnologia Agroindustrial, Universidade Federal de Pelotas

Titulação: Bacharel em Química Industrial de Alimentos

Área de Atuação: Ciência e Tecnologia dos Alimentos

Endereço completo para correspondência (bairro, cidade, estado, país e CEP): Rua Barão de Santa Tecla, 816, ap 103, centro, Pelotas, RS, Brasil, Cep: 96010-140. 
Telefones para contato: (53) 3275-7585

e-mail: preichardt_leidi@yahoo.com.br

Nome completo: Claire Tondo Vendruscolo

Filiação institucional: Universidade Federal de Pelotas

Função ou cargo ocupado: Professor Adjunto

Titulação: Doutorado em Engenharia de Alimentos pela Universidade Estadual de Campinas

Área de Atuação: Ciência e Tecnologia dos Alimentos

Endereço completo para correspondência (bairro, cidade, estado, país e CEP): Centro de Biotecnologia - Campus Universitário, s/nº, Caixa Postal 354, Pelotas, RS, Cep: 96010-900.

Telefones para contato: (53) 3275-7585

e-mail: claire@ufpel.tche.br

Nome completo: Márcia Arocha Gularte

Filiação institucional: Universidade Federal de Pelotas

Função ou cargo ocupado: Professor Adjunto

Titulação: Doutorado em Ciência e Tecnologia Agroindustrial pela Universidade Federal de Pelotas Área de Atuação: Ciência e Tecnologia dos Alimentos

Endereço completo para correspondência (bairro, cidade, estado, país e CEP): Departamento de Ciência dos Alimentos - Campus Universitário, s/nº, Caixa Postal 354, Pelotas, RS, Cep: 96010 900.

Telefones para contato: (53) 3275-7285

e-mail:gularte@ufpel.edu.br

Nome completo: Angelita da Silveira Moreira

Filiação institucional: Universidade Federal de Pelotas

Função ou cargo ocupado: Professor Adjunto

Titulação: Doutorado Biotecnologia Agricola pela Universidade Federal de Pelotas

Área de Atuação: Microbiologia Industrial e de Fermentações

Endereço completo para correspondência (bairro, cidade, estado, país e CEP): Centro de Biotecnologia - Campus Universitário, s/nº Caixa Postal 354, Pelotas, RS, Cep: 96010-900.

Telefones para contato: (53) 3275-7585

e-mail:angelita70@terra.com.br 